\title{
Genetic variations in the FADS1, FADS2 genes influence the proportions of long-chain $n-6$ polyunsaturated fatty acids in erythrocyte and plasma lipids
}

\author{
M. Al-Hilal and T. A. B. Sanders \\ Diabetes and Nutritional Sciences Division, King's College London, Franklin-Wilkins Building, 150 Stamford Street, \\ London SE1 9NH, UK
}

The capacity to convert linoleic acid (18:2n-6) into longer chain metabolites may be influenced by single nucleotide polymorphism (SNP) for fatty acid desaturase genes $F A D S 1$ and FADS 2 which encode delta-5 and delta- 6 desaturase enzymes ${ }^{(1)}$. We report the effects of rs 174537 and rs 174561 SNPs in the FADS1 and FADS2 respectively on the proportions of long-chain $n$ - 6 polyunsaturated fatty acids (PUFA) in plasma lipids and erythrocyte membrane phosphoglycerides in 123 men and 195 women aged $45-70$ years who were recruited into the MARINA study ${ }^{(2)}$. DNA was extracted from buffy coats and rs174537 and rs174561 genotypes were determined by KBiosciences UK. Plasma fatty acid composition and erythrocyte phosphoglycerides were determined by capillary gas liquid chromatography. The proportions (wt \%) of $n$-6 PUFA in erythrocytes (RBC) and plasma by genotype adjusted for age, gender, BMI and ethnicity are shown in the Table 1.

\begin{tabular}{|c|c|c|c|c|c|}
\hline & & \multicolumn{3}{|c|}{ rs174537 (FADS1) alleles } & \multirow[b]{2}{*}{$P$ value } \\
\hline & & GG $(n$ 151) & GT $(n$ 125) & TT $(n$ 30) & \\
\hline \multirow[t]{2}{*}{$20: 3 n-6$} & RBC & $1.71(1.66,1.76)$ & $1.99(1.93,2.05)$ & $2.24(2.12,2.36)$ & $8 \times 10^{-18}$ \\
\hline & Plasma & $1.54(1.49,1.58)$ & $1.68(1.63,1.73)$ & $1.79(1.67,1.90)$ & $6 \times 10^{-6}$ \\
\hline \multirow[t]{2}{*}{$20: 4 n-6$} & RBC & $16.92(16.60,17.25)$ & $16.59(16.21,16.96)$ & $16.01(15.29,16.73)$ & 0.06 \\
\hline & Plasma & $7.62(7.40,7.84)$ & $6.41(6.16,6.65)$ & $5.71(5.22,6.21)$ & $4 \times 10^{-16}$ \\
\hline \multirow[t]{4}{*}{$22: 4 n-6$} & RBC & $2.95(2.72,3.18)$ & $2.89(2.63,3.15)$ & $2.62(2.11,3.12)$ & 0.49 \\
\hline & Plasma & $0.60(0.58,0.62)$ & $0.57(0.54,0.59)$ & $0.51(0.46,0.56)$ & 0.004 \\
\hline & & \multicolumn{4}{|c|}{ rs174561 (FADS2) alleles } \\
\hline & & TT (n 162) & TC $(n$ 116) & CC (n 25) & \\
\hline \multirow[t]{2}{*}{$20: 3 n-6$} & RBC & $1.70(1.65,1.75)$ & $2.02(1.96,2.07)$ & $2.29(2.17,2.41)$ & $2.1 \times 10^{-20}$ \\
\hline & Plasma & $1.54(1.49,1.58)$ & $1.68(1.63,1.74)$ & $1.77(1.65,1.89)$ & $1 \times 10^{-5}$ \\
\hline \multirow[t]{2}{*}{$20: 4 n-6$} & RBC & $16.97(16.65,17.29)$ & $16.30(15.90,16.70)$ & $16.03(15.23,16.83)$ & 0.01 \\
\hline & Plasma & $7.55(7.34,7.76)$ & $6.28(6.03,6.52)$ & $5.75(5.22,6.29)$ & $6.1 \times 10^{-16}$ \\
\hline \multirow[t]{2}{*}{$22: 4 n-6$} & RBC & $2.97(2.74,3.19)$ & $2.84(2.57,3.12)$ & $2.67(2.12,3.22)$ & 0.551 \\
\hline & Plasma & $0.60(0.57,0.62)$ & $0.57(0.54,0.60)$ & $0.53(0.47,0.58)$ & 0.06 \\
\hline
\end{tabular}

Adjusted mean values with $95 \%$ CI. $P$ value is from ANCOVA adjusted for age, BMI, gender and ethnicity.

Carriers of the minor allele of the FADS1 SNP (rs174537) had higher proportions of dihomogammalinolenic acid (20:3n-6) in both RBC and plasma. The proportions of arachidonic acid (20:4n-6) and adrenic acid (22:4n-6) were corresponding lower in plasma but not in RBC lipids. Carriers of the minor allele of the FADS2 SNP (rs174561) also showed higher proportion of 20:3n-6 and a lower proportion of 20:4n-6 in plasma and RBC lipid. Previous reports of variations in the FADS1 and FADS2 genes have been mainly in children and confined to plasma phospholipids ${ }^{(1)}$. Our results show that older adults who carry the minor alleles of rs174537 and rs174561 have about $25 \%$ less 20:4n-6 in their plasma lipids than non-carriers. However, the impact of genotype on the proportion of 20:4n-6 in membrane lipids is much smaller presumably because acyltransferases can select the fatty acids required. The higher proportion of $20: 3 n-6$ in carriers of the minor alleles may be advantageous in that eicosanoid metabolites are less inflammatory and prothrombotic compared with those derived from $20: 4 n-6$.

1. Glaser C, Lattka E, Rzehak P, Steer C \& Koletzko B (2011) Genetic variation in polyunsaturated fatty acid metabolism and its potential relevance for human development and health. Matern Child Nutr. Suppl 2:27-40.

2. Sanders TA, Hall WL, Maniou Z, Lewis F, Seed PT \& Chowienczyk PJ (2011) Effect of low doses of long-chain n-3 PUFAs on endothelial function and arterial stiffness: a randomized controlled trial. Am J Clin Nutr. 94, 973-80. 